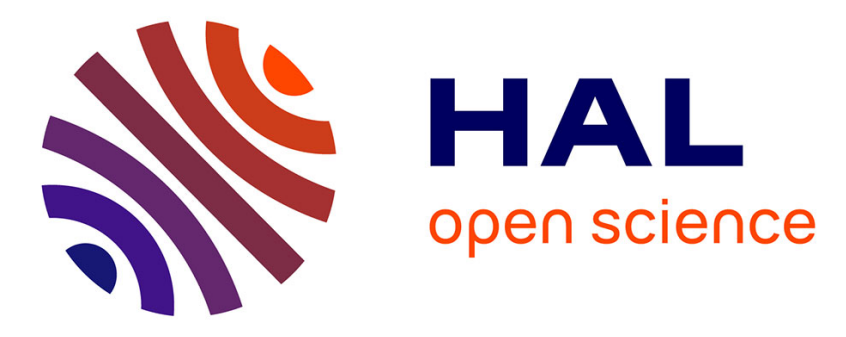

\title{
Blue-luminescent poly(p-phenylenevinylene) derivatives Synthesis and effect of side-group size on the optical properties
}

Nejmeddine Jaballah, Mejed Chemli, Khaled Hriz, Jean-Louis Fave, Mohamed Jouini, Mustapha Majdoub

\section{To cite this version:}

Nejmeddine Jaballah, Mejed Chemli, Khaled Hriz, Jean-Louis Fave, Mohamed Jouini, et al.. Blueluminescent poly(p-phenylenevinylene) derivatives Synthesis and effect of side-group size on the optical properties. European Polymer Journal, 2011, 47 (1), pp.78-87. 10.1016/j.eurpolymj.2010.10.017 . hal-01236802

\section{HAL Id: hal-01236802 \\ https://hal.science/hal-01236802}

Submitted on 2 Sep 2021

HAL is a multi-disciplinary open access archive for the deposit and dissemination of scientific research documents, whether they are published or not. The documents may come from teaching and research institutions in France or abroad, or from public or private research centers.
L'archive ouverte pluridisciplinaire HAL, est destinée au dépôt et à la diffusion de documents scientifiques de niveau recherche, publiés ou non, émanant des établissements d'enseignement et de recherche français ou étrangers, des laboratoires publics ou privés.

\section{(c)(1)}

Distributed under a Creative Commons Attribution| 4.0 International License 


\title{
Blue-luminescent poly( $p$-phenylenevinylene) derivatives: Synthesis and effect of side-group size on the optical properties
}

\author{
Nejmeddine Jaballah ${ }^{a}$, Mejed Chemli ${ }^{a}$, Khaled Hriz ${ }^{a}$, Jean-Louis Fave ${ }^{b}$, Mohamed Jouini ${ }^{c}$, \\ Mustapha Majdoub ${ }^{\mathrm{a}, *}$ \\ a Laboratoire des Polymères, Biopolymères et Matériaux Organiques (LPBMO), Faculté des Sciences de Monastir, Bd. de l'Environnement, 5019 Monastir, Tunisia \\ ${ }^{\mathrm{b}}$ INSP, Université Paris 6, CNRS UMR 7588, 140 rue de Lourmel, 75015 Paris, France \\ 'Laboratoire ITODYS, Université Paris 7, CNRS UMR 7086, Bâtiment Lavoisier, 15 rue Jean-Antoine de Baïf, 75205 Paris Cedex 13, France
}

\begin{abstract}
New conjugated polymers based on separated PPV-type chromophores and incorporating different types of solubilizing side-groups (ethoxy: P1, hexyloxy: P2, dodecyloxy: P3 and benzyloxy: P4) were synthesized via Wittig polycondensation, using a series of bisphenol A-derived di(triphenylphosphonium) salts as starting monomers. The polymers are soluble in common organic solvents and their structures were confirmed by ${ }^{1} \mathrm{H}$ NMR, ${ }^{13} \mathrm{C}$ NMR and FTIR spectroscopies. The optical properties of these materials were investigated by UV-vis absorption and fluorescence spectroscopies. In dilute solution, quasi-identical fluorescence spectra were obtained and all the polymers showed a blue emission $(420,445 \mathrm{~nm})$ and a narrow spectrum. In thin solid films, the polymers show side-group-dependent optical behavior and, whereas the emission remains blue in the case of P2, P3 and P4, a green fluo-rescence was observed for the ethoxylated polymer P1. From cyclic voltammetry analysis, the electrochemical band gaps were estimated to be 2.99, $3.07,3.15$ and 3.06 eV for P1, P2, P3 and P4, respectively. Single-layer diode devices of the [indium tin oxide/polymer/alumi-num] configuration have been fabricated and show relatively low turn-on voltages between 2.6 and $4.9 \mathrm{~V}$.
\end{abstract}

\section{Introduction}

The discovery of electroluminescence in poly( $p$-phenylenevinylene) (PPV) 20 years ago [1] created a new area in organic polymer applications. Since then, enormous progress has been made in the molecular engineering of $\pi$-conjugated polymers and in their uses as active materials in polymeric light-emitting diodes (PLEDs) [2-4]. These polymers are promising organic analogs of inorganic semi-conducting materials, and their applications to other devices, such as light-emitting electrochemical cells $[5,6]$, thin-film transistors [7], solar cells [8,9] and chemical sensors $[10,11]$ are currently expanding. The main advantages

\footnotetext{
* Corresponding author. Tel.: +216 73500 280; fax: +216 73500278 .

E-mail address: mustapha.majdoub@fsm.rnu.tn (M. Majdoub).
}

of using such semi-conducting polymers lie in their low cost and easy processibility. These materials are in fact compatible with solution processing techniques, thereby eliminating the expensive lithography and vacuum deposition steps required for the elaboration of inorganic semiconducting films. Solution processing also expands the repertoire of tolerant substrates and processing options, allowing flexible plastics to be used in conjunction with relatively simple methods such as spin coating, stamping and inkjet printing. However, an essential characteristic of these functional polymers is that their opto-electronic properties can be tuned, benefiting from the richness of organic synthesis and therefore from an adjustable macromolecular structure $[3,4]$.

Full color display applications require red, green, and blue emission, and whilst all three colors have been demonstrated in conjugated polymers, there are relatively 
few blue-emitting ones [12]. The first blue PLED was made from poly(9,9-dihexylfluorene) [13]. Other blue-electroluminescent polymers have since been reported, including 9,9-disubstituted polyfluorenes [14], polyphenylene derivatives [15], 3,4-disubstituted polythiophenes [16] and poly( $m$-phenylenevinylene) derivatives [17]. As regards, PPV-type polymers, a blue emission can be obtained by a partial conversion of the precursor polymer in the sulfonium route to PPV, giving a high concentration of interruptions in the conjugated chain $[18,19]$. In a second approach, introducing co-monomers with flexible linker groups into a step-growth condensation route to PPV can lead to blue-emitting copolymer [20]. However, these two methods only allow limited control of the length and distribution of the conjugated segments. Hence, emission occurs usually from the more highly conjugated segments, and the emission spectrum is therefore broadened and redshifted. To solve this problem, the confinement of the conjugation into a well defined and uniform length is one of the most successful strategies developed [3]. Some blueemitting polymers, incorporating uniform oligomeric PPV units which are separated by non-conjugated aliphatic spacer units in the polymer backbone, were reported $[21,22]$. Such polymers are homogeneous in terms of conjugation length and show narrow emission spectra and high photoluminescence quantum yields in the solid state, due to exciton confinement and reduction of luminescence loss via the quenching processes caused by $\pi-\pi$ stacking of the conjugated moieties [23]. Nevertheless, the relatively long aliphatic segments, used as spacers in these polymers, can act as barriers to injection and mobility of the charge carriers, resulting in higher turn-on voltages [2]. In our contribution to the molecular engineering of blue-emitting PPVs, we follow the same confined conjugation strategy, but using shorter spacer groups. In our previous work, we reported a confined PPV-type polymer derived from bisphenol A (Scheme 1, P1) [24]. This polymer incorporates a distyrylbenzene (DSB) unit as chromophore, an isopropylidene group as spacer unit and an ethoxy side-chain as solubilizing group. It showed a low operating voltage and a blue emission with a narrow fluorescence spectrum in dilute solution. However, in a thin solid film the emission was red-shifted and the spectrum was significantly broadened, due to $\pi-\pi$ stacking of the DSB segments. Herein, we follow the same approach and report on the design, synthesis and characterization of a series of P1 analogs incorporating different types of solubilizing side-groups. The effect of the side-group structure on the optical properties, and especially on the $\pi-\pi$ interaction in the polymer solid film, was investigated.

\section{Experimental}

\subsection{Materials and measurements}

All starting materials were purchased from Acros Organics. Tetrahydrofuran (THF) was dried over Na/benzophenone and freshly distilled before use. Acetone was stirred over and distilled from potassium carbonate under argon. ${ }^{1} \mathrm{H}$ NMR and ${ }^{13} \mathrm{C}$ NMR spectral data were obtained on a Bruker AV 300 spectrometer and the chemical shifts were referenced to the $\mathrm{CHCl}_{3}$ signal (7.26 ppm for ${ }^{1} \mathrm{H}, 77.16 \mathrm{ppm}$ for ${ }^{13} \mathrm{C}$ ). The FTIR spectra were acquired on a Perkin-Elmer BX FTIR system spectrometer (by dispersing samples in $\mathrm{KBr}$ disks) and the UV-vis absorption spectra on a Cary 2300 spectrophotometer. Fluorescence spectra were obtained on a Jobin-Yvon spectrometer HR460 coupled to a nitrogen-cooled Si charged-coupled device (CCD) detector; samples were excited at $365 \mathrm{~nm}$ with a $450 \mathrm{~W}$ xenon lamp. For optical solid-state measurements, the polymer films were spin-cast onto quartz substrates from chloroform solutions. Cyclic voltammetry (CV) was performed on an EG\&G model
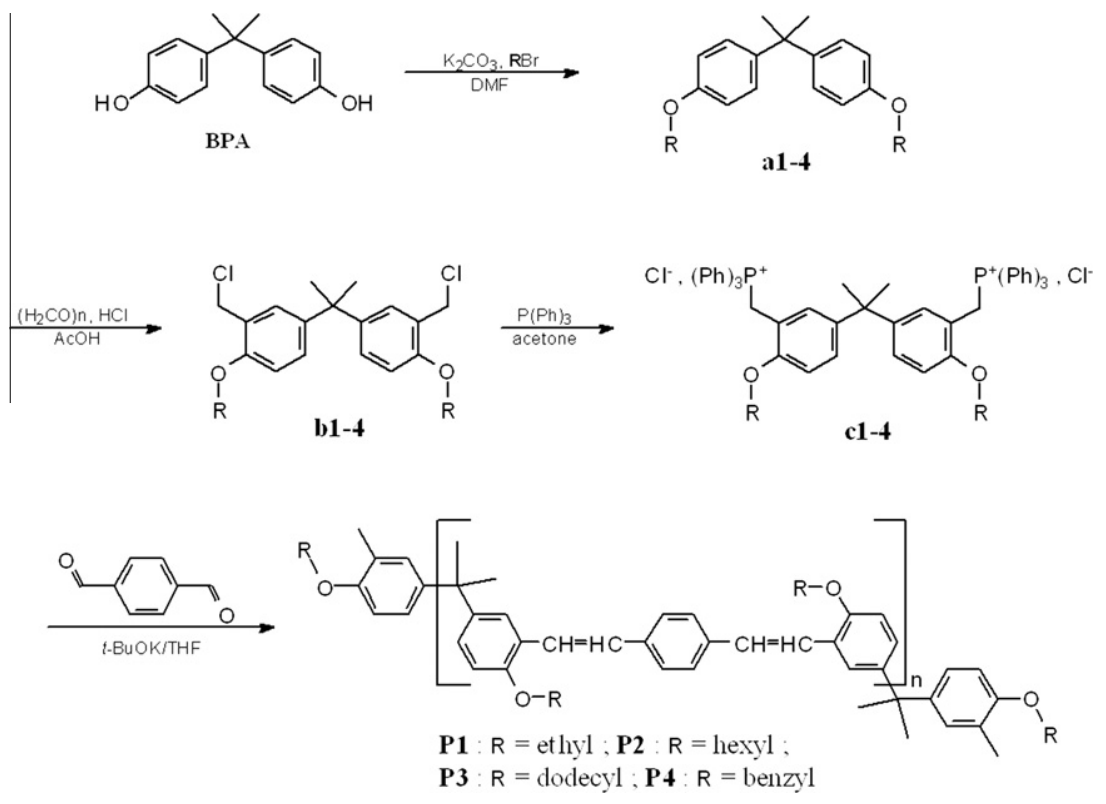

Scheme 1. Synthetic route to polymers P1-4. 
273 potentiostat/galvanostat (Princeton Applied Research) in a three-electrode cell using polymer film that was dropcast onto an indium tin oxide (ITO) working electrode. The measurements were carried out at a scan rate of $50 \mathrm{mV} \mathrm{s}^{-1}$ and referenced to a saturated calomel electrode (SCE), using $0.1 \mathrm{M}$ tetrabutylammonium perchlorate $\left((\mathrm{n}-\mathrm{Bu})_{4} \mathrm{NClO}_{4}\right)$ in acetonitrile as supporting electrolyte. The measurements were performed at room temperature and the cell was briefly deoxygenated with argon before each reductive scan.

\subsection{Preparation of bisphenol A (BPA) ethers (a1-4)}

A mixture of BPA ( $2.28 \mathrm{~g}, 10 \mathrm{mmol})$, potassium carbonate $(3.48 \mathrm{~g}, 25 \mathrm{mmol})$ and the appropriate alkyl bromide (25 mmol) were stirred in $20 \mathrm{ml}$ of DMF at $60{ }^{\circ} \mathrm{C}$ (except in the case of $\mathbf{a 1}$, at room temperature). After $8 \mathrm{~h}$, the reaction mixture was poured into distilled water and extracted with dichloromethane. The extract was washed with distilled water, dried over anhydrous $\mathrm{MgSO}_{4}$ and concentrated at reduced pressure. The resultant crude product was purified by recrystallization from an appropriate solvent.

a1: $\mathrm{R}=$ ethyl; recrystallization from ethanol/water (3/ 1); aspect: white needle-like crystals; yield: 95\%; m.p. $48{ }^{\circ} \mathrm{C}$; ${ }^{1} \mathrm{H}$ NMR $\left(300 \mathrm{MHz}, \mathrm{CDCl}_{3}, \delta\right): 7.13$ (d, $J=8.7 \mathrm{~Hz}$, $4 \mathrm{H}, \mathrm{Ar}-\mathrm{H}), 6.80(\mathrm{~d}, J=8.7 \mathrm{~Hz}, 4 \mathrm{H}, \mathrm{Ar}-\mathrm{H}), 4.00(\mathrm{q}, J=6.9 \mathrm{~Hz}$, $\left.4 \mathrm{H}, \mathrm{OCH}_{2}\right), 1.64\left(\mathrm{~s}, 6 \mathrm{H}, \mathrm{C}\left(\mathrm{CH}_{3}\right)_{2}\right), 1.40(\mathrm{t}, J=6.9 \mathrm{~Hz}, 6 \mathrm{H}$, $\left.\mathrm{CH}_{3}\right) ;{ }^{13} \mathrm{C}$ NMR $\left(75.5 \mathrm{MHz}, \mathrm{CDCl}_{3}, \delta\right): 156.8,143.1,127.8$, 113.9, 63.4, 41.8, 31.2, 15.1; FTIR $\left(\mathrm{cm}^{-1}\right): 1247$ (s, C-O-C asymmetric stretching), 1049 (m, C-O-C symmetric stretching).

a2: $\mathrm{R}=$ hexyl; recrystallization from ethanol; aspect: white crystals; yield: $90 \%$; m.p. $29{ }^{\circ} \mathrm{C}$; ${ }^{1} \mathrm{H}$ NMR $(300 \mathrm{MHz}$, $\left.\mathrm{CDCl}_{3}, \delta\right): 7.16(\mathrm{~d}, J=8.7 \mathrm{~Hz}, 4 \mathrm{H}, \mathrm{Ar}-\mathrm{H}), 6.82(\mathrm{~d}, J=8.7 \mathrm{~Hz}$, $4 \mathrm{H}, \mathrm{Ar}-\mathrm{H}), 3.95\left(\mathrm{t}, J=6.6 \mathrm{~Hz}, 4 \mathrm{H}, \mathrm{OCH}_{2}\right), 1.79\left(\mathrm{tt}, J_{1}=J_{2}=\right.$ $\left.6.6 \mathrm{~Hz}, 4 \mathrm{H},-\mathrm{OCH}_{2} \mathrm{CH}_{2}-\right), 1.66\left(\mathrm{~s}, 6 \mathrm{H}, \mathrm{C}\left(\mathrm{CH}_{3}\right)_{2}\right), 1.55-1.30$ $\left(\mathrm{m}, 12 \mathrm{H},-\left(\mathrm{CH}_{2}\right)_{3}-\right), 0.94\left(\mathrm{t}, J=6.6 \mathrm{~Hz}, 6 \mathrm{H}, \mathrm{CH}_{3}\right) ;{ }^{13} \mathrm{C} \mathrm{NMR}$ $\left(75.5 \mathrm{MHz}, \mathrm{CDCl}_{3}, \delta\right): 157.0,143.0,127.8,113.9,68.0$, 41.7, 31.7, 31.2, 29.4, 25.9, 22.8, 14.2; FTIR $\left(\mathrm{cm}^{-1}\right): 1249$ (s, C-O-C asymmetric stretching), 1032 (m, C-O-C symmetric stretching).

a3: $\mathrm{R}=$ dodecyl; recrystallization from ethanol; aspect: white powder; yield: $94 \%$; m.p. $52{ }^{\circ} \mathrm{C}$; ${ }^{1} \mathrm{H}$ NMR $(300 \mathrm{MHz}$, $\left.\mathrm{CDCl}_{3}, \delta\right): 7.14(\mathrm{~d}, J=8.7 \mathrm{~Hz}, 4 \mathrm{H}, \mathrm{Ar}-\mathrm{H}), 6.80(\mathrm{~d}, J=8.7 \mathrm{~Hz}$, $4 \mathrm{H}, \quad \mathrm{Ar}-\mathrm{H}), 3.93\left(\mathrm{t}, J=6.6 \mathrm{~Hz}, 4 \mathrm{H}, \mathrm{OCH}_{2}\right), 1.77$ (tt, $\left.J_{1}=J_{2}=6.6 \mathrm{~Hz}, 4 \mathrm{H},-\mathrm{OCH}_{2} \underline{\mathrm{C}}_{2}-\right), 1.65\left(\mathrm{~s}, 6 \mathrm{H}, \mathrm{C}\left(\mathrm{CH}_{3}\right)_{2}\right)$, 1.55-1.20 (m, 36H, $\left.-\left(\mathrm{CH}_{2}\right)_{9}{ }^{-}\right), 0.90\left(\mathrm{t}, J=6.6 \mathrm{~Hz}, 6 \mathrm{H}, \mathrm{CH}_{3}\right)$; ${ }^{13} \mathrm{C}$ NMR $\left(75.5 \mathrm{MHz}, \mathrm{CDCl}_{3}, \delta\right): 157.0,143.0,127.8,113.9$, 68.0, 41.7, 32.1, 31.2, 29.8, 29.7, 29.6, 26.2, 22.8, 14.3; FTIR $\left(\mathrm{cm}^{-1}\right): 1244$ (s, C-O-C asymmetric stretching), $1026(\mathrm{~m}$, C-O-C symmetric stretching).

a4: $\mathrm{R}$ = benzyl; recrystallization from acetone; aspect: white crystals; yield: 93\%; m.p. $128{ }^{\circ} \mathrm{C} ;{ }^{1} \mathrm{H} \quad \mathrm{NMR}$ (300 $\left.\mathrm{MHz}, \mathrm{CDCl}_{3}, \delta\right): 7.50-7.32(\mathrm{~m}, 10 \mathrm{H}, \mathrm{Ar}-\mathrm{H}$ of benzyl group) $7.20(\mathrm{~d}, J=8.7 \mathrm{~Hz}, 4 \mathrm{H}, \operatorname{Ar}-\mathrm{H}), 6.93(\mathrm{~d}, J=8.7 \mathrm{~Hz}$, $4 \mathrm{H}, \mathrm{Ar}-\mathrm{H}), 5.07\left(\mathrm{~s}, 4 \mathrm{H}, \mathrm{OCH}_{2}\right), 1.68\left(\mathrm{~s}, 6 \mathrm{H}, \mathrm{C}\left(\mathrm{CH}_{3}\right)_{2}\right) ;{ }^{13} \mathrm{C}$ NMR $\left(75.5 \mathrm{MHz}, \mathrm{CDCl}_{3}, \delta\right): 156.8,143.5,137.3,128.7$, 128.0, 127.9, 127.7, 114.3, 70.1, 41.8, 31.2; FTIR $\left(\mathrm{cm}^{-1}\right)$ : 1240 (s, C-O-C asymmetric stretching), 1014 (m, C-O-C symmetric stretching).

\subsection{Synthesis of dichloromethyl derivatives (b1-4)}

A mixture of the corresponding BPA ether (a1-4) (10 mmol), paraformaldehyde $\left(2.50 \mathrm{~g}, 80 \mathrm{mmol}\right.$ of $\left.\mathrm{CH}_{2} \mathrm{O}\right)$, and $37 \%$ aqueous $\mathrm{HCl}(8.5 \mathrm{ml}, 102 \mathrm{mmol})$ in $30 \mathrm{ml}$ of acetic acid was stirred at $60^{\circ} \mathrm{C}$. The progress of the reaction was monitored by TLC ( $\mathrm{R}=$ ethyl, hexyl or benzyl; eluent: hexane/ethyl acetate $6 / 1$ ) or by ${ }^{1} \mathrm{H}$ NMR analysis ( $\mathrm{R}=$ dodecyl). The resulting mixture was then poured into distilled water and extracted with dichloromethane. The organic layer was washed several times with distilled water, dried over anhydrous $\mathrm{MgSO}_{4}$ and concentrated at reduced pressure. The resultant crude product was purified by recrystallization from an appropriate solvent.

b1: $\mathrm{R}=$ ethyl; reaction time: $4 \mathrm{~h}$; recrystallization from hexane; aspect: colorless crystals; yield: $75 \%$; m.p. $80^{\circ} \mathrm{C}$; ${ }^{1} \mathrm{H}$ NMR (300 MHz, $\mathrm{CDCl}_{3}, \delta$ ): 7.21 (d, $J=2.7,2 \mathrm{H}, \mathrm{Ar}-\mathrm{H}$ ), $7.10\left(\mathrm{dd}, J_{1}=2.7 \mathrm{~Hz}, J_{2}=8.4 \mathrm{~Hz}, 2 \mathrm{H}, \operatorname{Ar}-\mathrm{H}\right), 6.77(\mathrm{~d}$, $J=8.4 \mathrm{~Hz}, 2 \mathrm{H}, \mathrm{Ar}-\mathrm{H}), 4.63\left(\mathrm{~s}, 4 \mathrm{H}, \mathrm{CH}_{2} \mathrm{Cl}\right), 4.07$ (q, $J=6.9 \mathrm{~Hz}$ $\left.4 \mathrm{H}, \mathrm{OCH}_{2}\right), 1.64\left(\mathrm{~s}, 6 \mathrm{H}, \mathrm{C}\left(\mathrm{CH}_{3}\right)_{2}\right), 1.43(\mathrm{t}, J=6.9 \mathrm{~Hz}, 6 \mathrm{H}$ $\left.\mathrm{CH}_{3}\right) ;{ }^{13} \mathrm{C}$ NMR $\left(75.5 \mathrm{MHz}, \mathrm{CDCl}_{3}, \delta\right): 154.9,142.7,129.0$ $128.5,125.3,111.4,64.0,42.2,41.8,31.1,15.0$; FTIR $\left(\mathrm{cm}^{-1}\right): 603$ (s, C-Cl stretching).

b2: $\mathrm{R}=$ hexyl; reaction time: $10 \mathrm{~h}$; aspect: colorless viscous liquid; yield: $65 \%$; ${ }^{1} \mathrm{H}$ NMR $\left(300 \mathrm{MHz}, \mathrm{CDCl}_{3}, \delta\right.$ ): 7.21 $(\mathrm{d}, J=2.1 \mathrm{~Hz}, 2 \mathrm{H}, \mathrm{Ar}-\mathrm{H}), 7.11\left(\mathrm{dd}, J_{1}=2.1 \mathrm{~Hz}, J_{2}=8.7 \mathrm{~Hz}, 2 \mathrm{H}\right.$ $\mathrm{Ar}-\mathrm{H}), 6.76(\mathrm{~d}, \mathrm{~J}=8.7 \mathrm{~Hz}, 2 \mathrm{H}, \mathrm{Ar}-\mathrm{H}), 4.62\left(\mathrm{~s}, 4 \mathrm{H}, \mathrm{CH}_{2} \mathrm{Cl}\right), 3.99$ $\left(\mathrm{t}, J=6.6 \mathrm{~Hz}, 4 \mathrm{H}, \mathrm{OCH}_{2}\right), 1.76\left(\mathrm{tt}, J_{1}=J_{2}=6.6 \mathrm{~Hz}, 4 \mathrm{H}\right.$, $\left.\mathrm{OCH}_{2} \mathrm{CH}_{2}-\right), 1.64\left(\mathrm{~s}, 6 \mathrm{H}, \mathrm{C}\left(\mathrm{CH}_{3}\right)_{2}\right), 1.55-1.26(\mathrm{~m}, 12 \mathrm{H},-$ $\left.\left(\mathrm{CH}_{2}\right)_{3}{ }^{-}\right), 0.91\left(\mathrm{t}, J=6.6 \mathrm{~Hz}, 6 \mathrm{H}, \mathrm{CH}_{3}\right) ;{ }^{13} \mathrm{C} \mathrm{NMR}(75.5 \mathrm{MHz}$, $\left.\mathrm{CDCl}_{3}, \delta\right): 155.0,142.6,128.9,128.4,125.2,111.2,68.3$, 42.2, 41.8, 31.6, 31.1, 29.3, 25.9, 22.7, 14.1; FTIR $\left(\mathrm{cm}^{-1}\right)$ : 602 (s, C-Cl stretching).

b3: $\mathrm{R}=$ dodecyl; reaction time: $76 \mathrm{~h}$; recrystallization from ethanol; aspect: white powder, yield: 56\%; m.p. $48{ }^{\circ} \mathrm{C}$; ${ }^{1} \mathrm{H}$ NMR $\left(300 \mathrm{MHz}, \mathrm{CDCl}_{3}, \delta\right): 7.21(\mathrm{~d}, J=2.1 \mathrm{~Hz}$, $2 \mathrm{H}, \mathrm{Ar}-\mathrm{H}$ ), 7.11 (dd, $J_{1}=2.1 \mathrm{~Hz}, J_{2}=8.7 \mathrm{~Hz}, 2 \mathrm{H}, \mathrm{Ar}-\mathrm{H}$ ), 6.76 (d, $J=8.7 \mathrm{~Hz}, 2 \mathrm{H}, \mathrm{Ar}-\mathrm{H}), 4.63\left(\mathrm{~s}, 4 \mathrm{H}, \mathrm{CH}_{2} \mathrm{Cl}\right), 3.99(\mathrm{t}$, $\left.J=6.3 \mathrm{~Hz}, \quad 4 \mathrm{H}, \quad \mathrm{OCH}_{2}\right), 1.81\left(\mathrm{tt}, J_{1}=J_{2}=6.3 \mathrm{~Hz}, 4 \mathrm{H}\right.$, $\left.\mathrm{OCH}_{2} \mathrm{CH}_{2}-\right), 1.64\left(\mathrm{~s}, 6 \mathrm{H}, \mathrm{C}\left(\mathrm{CH}_{3}\right)_{2}\right), 1.55-1.20(\mathrm{~m}, 36 \mathrm{H},-$ $\left.\left(\mathrm{CH}_{2}\right)_{9-}\right), 0.89\left(\mathrm{t}, J=6.3 \mathrm{~Hz}, 6 \mathrm{H}, \mathrm{CH}_{3}\right) ;{ }^{13} \mathrm{C} \mathrm{NMR}(75.5 \mathrm{MHz}$, $\left.\mathrm{CDCl}_{3}, \delta\right): 155.0,142.7,129.0,128.5,125.2,111.2,68.3$ $42.3,41.8,32.1,31.2,29.8,29.8,29.7,29.5,29.4,26.2$, 22.8, 14.3; FTIR $\left(\mathrm{cm}^{-1}\right)$ : 620 (s, C-Cl stretching).

b4: $\mathrm{R}=$ benzyl; reaction time: $50 \mathrm{~h}$; recrystallization from ethanol; aspect: white powder, yield: 75\%, m.p. $143{ }^{\circ} \mathrm{C} ;{ }^{1} \mathrm{H}$ NMR $\left(300 \mathrm{MHz}, \mathrm{CDCl}_{3}, \delta\right): 7.50-7.31(\mathrm{~m}, 10 \mathrm{H}$ Ar-H of benzyl group), 7.24 (d, $J=2.4 \mathrm{~Hz}, 2 \mathrm{H}, \mathrm{Ar}-\mathrm{H}$ ), 7.11 (dd, $\left.J_{1}=2.4 \mathrm{~Hz}, J_{2}=8.7 \mathrm{~Hz}, 2 \mathrm{H}, \mathrm{Ar}-\mathrm{H}\right), 6.84(\mathrm{~d}, J=8.7 \mathrm{~Hz}$, $2 \mathrm{H}, \mathrm{Ar}-\mathrm{H}), 5.11\left(\mathrm{~s}, 4 \mathrm{H}, \mathrm{OCH}_{2}\right), 4.67\left(\mathrm{~s}, 4 \mathrm{H}, \mathrm{CH}_{2} \mathrm{Cl}\right), 1.64(\mathrm{~s}$, $\left.6 \mathrm{H}, \mathrm{C}\left(\mathrm{CH}_{3}\right)_{2}\right) ;{ }^{13} \mathrm{C}$ NMR $\left(75.5 \mathrm{MHz}, \mathrm{CDCl}_{3}, \delta\right): 154.7,143.2$, $137.2,129.1,128.7,128.5,128.0,127.3,125.6,111.8$, 70.3, 42.3, 41.9, 31.1; FTIR $\left(\mathrm{cm}^{-1}\right): 602$ (s, C-Cl stretching)

\subsection{Synthesis of diphosphonium salts (c1-4)}

A solution of the dichloromethyl derivative (b1-4) $(10 \mathrm{mmol})$ and triphenylphosphine $(5.82 \mathrm{~g}, 22 \mathrm{mmol})$ in anhydrous acetone $(50 \mathrm{ml})$ was stirred and heated at reflux for $12 \mathrm{~h}$ in an argon atmosphere. The reaction mixture was then cooled; the resulting white precipitate filtered off, 
washed with diethyl ether several times and dried under vacuum.

c1: $\mathrm{R}=$ ethyl; yield: 92\%; m.p. $220{ }^{\circ} \mathrm{C} ;{ }^{1} \mathrm{H} \quad \mathrm{NMR}$ (300 MHz, $\mathrm{CDCl}_{3}, \delta$ ): 7.80-7.52 (m, 30H, $\left.\mathrm{P}(\mathrm{Ph})_{3}\right), 7.14$ (s, $2 \mathrm{H}, \mathrm{Ar}-\mathrm{H}), 7.02$ (d, J = 8.7 Hz, 2H, Ar-H), 6.49 (d, $J=8.7 \mathrm{~Hz}$, $2 \mathrm{H}, \mathrm{Ar}-\mathrm{H}$ ), $5.18\left(\mathrm{~d},{ }^{2} J_{\mathrm{H}-\mathrm{P}}=14.1 \mathrm{~Hz}, 4 \mathrm{H}, \mathrm{CH}_{2} \mathrm{P}\right), 3.47(\mathrm{q}$, $\left.J=6.9 \mathrm{~Hz}, 4 \mathrm{H}, \mathrm{OCH}_{2}\right), 1.20\left(\mathrm{~s}, 6 \mathrm{H}, \mathrm{C}\left(\mathrm{CH}_{3}\right)_{2}\right), 0.99(\mathrm{t}$, $\left.J=6.9 \mathrm{~Hz}, 6 \mathrm{H}, \mathrm{CH}_{3}\right) ;{ }^{13} \mathrm{C} \mathrm{NMR}\left(75.5 \mathrm{MHz}, \mathrm{CDCl}_{3}, \delta\right): 154.7$, 142.2, 135.0, $134.2(\mathrm{~d}, J=9.7 \mathrm{~Hz}), 131.5,130.2$ (d, $J=12.5 \mathrm{~Hz}), \quad 127.6, \quad 118.15$ (d, $J=85.5 \mathrm{~Hz}), 114.5$ (d, $J=9.0 \mathrm{~Hz}$ ), $110.8,63.38,41.1,30.4,24.9\left(\mathrm{~d},{ }^{1} J_{\mathrm{C}-\mathrm{P}}=47.8 \mathrm{~Hz}\right.$, $\left.\mathrm{CH}_{2} \mathrm{P}\right), 14.7$; FTIR $\left(\mathrm{cm}^{-1}\right)$ : 1112 (s, P-C stretching), 512 (s, $\mathrm{P}-\mathrm{Cl}$ stretching).

c2: $\quad \mathrm{R}=$ hexyl; yield: $85 \%$; m.p. $230{ }^{\circ} \mathrm{C} ;{ }^{1} \mathrm{H} \quad \mathrm{NMR}$ (300 MHz, $\left.\mathrm{CDCl}_{3}, \delta\right): 7.80-7.45\left(\mathrm{~m}, 30 \mathrm{H}, \mathrm{P}(\mathrm{Ph})_{3}\right), 7.08(\mathrm{~s}$, $2 \mathrm{H}, \mathrm{Ar}-\mathrm{H}$ ), 7.00 (d, $J=8.7 \mathrm{~Hz}, 2 \mathrm{H}, \mathrm{Ar}-\mathrm{H}), 6.47$ (d, $J=8.7 \mathrm{~Hz}$, $2 \mathrm{H}, \mathrm{Ar}-\mathrm{H}), 5.15\left(\mathrm{~d},{ }^{2} J_{\mathrm{H}-\mathrm{P}}=14.1 \mathrm{~Hz}, 4 \mathrm{H}, \mathrm{CH}_{2} \mathrm{P}\right), 3.37(\mathrm{t}$, $\left.J=6.6 \mathrm{~Hz}, 4 \mathrm{H}, \mathrm{OCH}_{2}\right), 1.40-1.10\left(\mathrm{~m}, 22 \mathrm{H},-\left(\mathrm{CH}_{2}\right)_{4}-\right.$ and $\left.\mathrm{C}\left(\mathrm{CH}_{3}\right)_{2}\right), 0.86\left(\mathrm{t}, J=6.6 \mathrm{~Hz}, 6 \mathrm{H}, \mathrm{CH}_{3}\right) ;{ }^{13} \mathrm{C} \mathrm{NMR}(75.5 \mathrm{MHz}$, $\left.\mathrm{CDCl}_{3}, \delta\right): 154.8,143.0,134.9,134.2(\mathrm{~d}, J=9.7 \mathrm{~Hz}), 131.4$, 130.1 (d, $J=12.5 \mathrm{~Hz}$ ), 127.5, $118.1(\mathrm{~d}, J=85.5 \mathrm{~Hz}$ ), 114.5 (d, $J=9.0 \mathrm{~Hz}$ ), 110.9, 67.8, 41.0, 31.6, 30.4, 29.0, 25.7, 25.4 (d, $\left.J=48.7 \mathrm{~Hz}, \mathrm{CH}_{2} \mathrm{P}\right), 22.6,14.11$; FTIR $\left(\mathrm{cm}^{-1}\right)$ : 1112 (s, $\mathrm{P}-\mathrm{C}$ stretching), 506 (s, P-Cl stretching).

c3: $\mathrm{R}=$ dodecyl; yield: $80 \%$; m.p. $176{ }^{\circ} \mathrm{C} ;{ }^{1} \mathrm{H}$ NMR (300 MHz. $\mathrm{CDCl}_{3} . \delta$ ): 7.80-7.45 (m, 30H, $\left.\mathrm{P}(\mathrm{Ph})_{3}\right), 7.11(\mathrm{~s}$, $2 \mathrm{H}, \operatorname{Ar}-\mathrm{H}$ ), 7.01 (d, $J=8.7 \mathrm{~Hz}, 2 \mathrm{H}, \mathrm{Ar}-\mathrm{H}), 6.48$ (d, $J=8.7 \mathrm{~Hz}$, $2 \mathrm{H}, \operatorname{Ar}-\mathrm{H}), 5.20\left(\mathrm{~d},{ }^{2} J_{\mathrm{H}-\mathrm{P}}=13.8 \mathrm{~Hz}, 4 \mathrm{H}, \mathrm{CH}_{2} \mathrm{P}\right), 3.38(\mathrm{t}$, $\left.J=6.3 \mathrm{~Hz}, 4 \mathrm{H}, \mathrm{OCH}_{2}\right), 1.40-1.00\left(\mathrm{~m}, 46 \mathrm{H},-\left(\mathrm{CH}_{2}\right)_{10^{-}}\right.$and $\left.\mathrm{C}\left(\mathrm{CH}_{3}\right)_{2}\right), 0.86\left(\mathrm{t}, J=6.6 \mathrm{~Hz}, 6 \mathrm{H}, \mathrm{CH}_{3}\right) ;{ }^{13} \mathrm{C} \mathrm{NMR}(75.5 \mathrm{MHz}$, $\left.\mathrm{CDCl}_{3}, \delta\right): 154.9,142.0,134.9,134.2(\mathrm{~d}, J=9.8 \mathrm{~Hz}), 131.5$, 130.1 (d, $J=12.2 \mathrm{~Hz}$ ), 127.6, 118.2 (d, $J=85.1 \mathrm{~Hz}), 114.5$ (d, $J=9.0 \mathrm{~Hz}), 110.8,67.8,41.1,32.0,30.4,29.8,29.7$, 29.5, 29.1, 26.1, 24.7 (d, $J=48.7 \mathrm{~Hz}, \mathrm{CH}_{2} \mathrm{P}$ ), 22.8, 14.3; FTIR $\left(\mathrm{cm}^{-1}\right)$ : 1113 (s, P-C stretching), 507 (s, P-Cl stretching).

c4: $\mathrm{R}=$ benzyl; yield: $80 \%$; m.p. $266^{\circ} \mathrm{C}$; ${ }^{1} \mathrm{H}$ NMR (300 MHz, $\left.\mathrm{CDCl}_{3}, \delta\right)$ : 7.75-7.40 (m, 30H, $\left.\mathrm{P}(\mathrm{Ph})_{3}\right), 7.40-$ $7.30(\mathrm{~m}, 6 \mathrm{H}, \mathrm{Ar}-\mathrm{H}), 7.17$ (s, 2H, Ar-H), 7.13-7.01 (m, 6H, $\mathrm{Ar}-\mathrm{H}), 6.60(\mathrm{~d}, J=8.7 \mathrm{~Hz}, 2 \mathrm{H}, \operatorname{Ar}-\mathrm{H}), 5.17$ (d, ${ }^{2} J_{\mathrm{H}-\mathrm{P}}=$ $\left.13.8 \mathrm{~Hz}, 4 \mathrm{H}, \mathrm{CH}_{2} \mathrm{P}\right), 4.49\left(\mathrm{~s}, 4 \mathrm{H}, \mathrm{OCH}_{2}\right), 1.23(\mathrm{~s}, 6 \mathrm{H}$, $\left.\mathrm{C}\left(\mathrm{CH}_{3}\right)_{2}\right) ;{ }^{13} \mathrm{C}$ NMR $\left(75.5 \mathrm{MHz}, \mathrm{CDCl}_{3}, \delta\right): 154.6,142.6$, 134.9, 134.2 (d, J=9.7 Hz), 133.7, 131.6, 130.0 (d, $J=12.5 \mathrm{~Hz}$ ), 128.8, 128.7, 128.4, 127.6, 127.4, 118.0 (d, $J=85.3 \mathrm{~Hz}), 115.2(\mathrm{~d}, J=8.9 \mathrm{~Hz}), 111.4,70.0,41.2,30.3$, $25.1\left(\mathrm{~d}, J=47.8 \mathrm{~Hz}, \mathrm{CH}_{2} \mathrm{P}\right)$; FTIR $\left(\mathrm{cm}^{-1}\right)$ : 1111 (s, P-C stretching), 505 (s, P-Cl stretching).

\subsection{Synthesis of the polymers (P1-4)}

Method A: To a stirred mixture of an equimolar amount of the terephthaldicarboxaldehyde $(0.135 \mathrm{~g}, 1 \mathrm{mmol})$ and the diphosphonium salt (c1-4) (1 mmol) in $10 \mathrm{ml}$ of anhydrous THF, $10 \mathrm{ml}$ of a $0.5 \mathrm{M} \mathrm{t}$-BuOK solution in THF ( $5 \mathrm{mmol}$ ) was added dropwise at room temperature under an argon atmosphere. The reaction mixture was stirred for $24 \mathrm{~h}$ after the addition and then acidified with $3 \%$ aqueous hydrochloric acid, poured into water and extracted with chloroform. The organic phase was washed with water, concentrated and then precipitated in methanol. The polymer was then filtered and dried under vacuum for $48 \mathrm{~h}$.
Method B: As described in Method A, with stirring $4 \mathrm{~h}$ at reflux $\left(60^{\circ} \mathrm{C}\right)$ after $24 \mathrm{~h}$ at room temperature.

P1: $\mathrm{R}=$ ethyl; aspect: yellow powder; ${ }^{1} \mathrm{H} \quad \mathrm{NMR}$ (300 MHz, $\mathrm{CDCl}_{3}, \delta$ ): 8.10-6.40 (br m, aromatic and vinylic $\mathrm{H})$, 4.10-3.70 ( $\left.\mathrm{m}, \mathrm{OCH}_{2}\right), 2.18\left(\mathrm{~s}, \mathrm{Ar}-\mathrm{CH}_{3}\right.$ terminal group), 1.70-1.50 (m, C $\left.\left(\mathrm{CH}_{3}\right)_{2}\right)$, $1.45-1.15\left(\mathrm{~m}, \mathrm{CH}_{3}\right) ;{ }^{13} \mathrm{C} \mathrm{NMR}$ $\left(75.5 \mathrm{MHz}, \mathrm{CDCl}_{3}, \delta\right): 155.1,154.5,143.0,142.5,137.3$, $136.8,129.8,129.3,128.7,127.5,127.1,126.9,126.2$, 125.8, 125.0, 124.8, 124.1, 111.8, 111.5, 110.4, 64.1, 63.6, 42.0; 41.7, 31.3, 31.0, 16.6, 15.1, 15.0; FTIR $\left(\mathrm{cm}^{-1}\right): 3020$ (w, aromatic and vinylic C-H stretching), 2973, 2928, 2872 (w, aliphatic C-H stretching), 1684, 1601 (m, C=C stretching), 1246 (s, C-O-C asymmetric stretching), 1043 (m, C-O-C symmetric stretching), 806 (s, aromatic $\mathrm{C}-\mathrm{H}$ out-of-plane bending), $967(\mathrm{~m}$, trans- $\mathrm{HC}=\mathrm{CH}$ out-of-plane bending), 865 ( $\mathrm{w}$, cis- $\mathrm{HC}=\mathrm{CH}$ out-of-plane bending).

P2: $\mathrm{R}=$ hexyl; aspect: yellow powder; ${ }^{1} \mathrm{H}$ NMR (300 MHz, $\mathrm{CDCl}_{3}, \delta$ ): 7.80-6.40 (br m, aromatic and vinylic $\mathrm{H})$, 4.15-3.80 ( $\left.\mathrm{m}, \mathrm{OCH}_{2}\right), 2.21$ ( $\mathrm{s}, \mathrm{Ar}-\mathrm{CH}_{3}$ terminal group), 2.00-1.20 (br m, $-\left(\mathrm{CH}_{2}\right)_{4}-$ and $\left.\mathrm{C}\left(\mathrm{CH}_{3}\right)_{2}\right), 0.93\left(\mathrm{~s}, \mathrm{CH}_{3}\right) ;{ }^{13} \mathrm{C}$ NMR $\left(75.5 \mathrm{MHz}, \mathrm{CDCl}_{3}, \delta\right): 155.3,154.8,142.9,142.5$, $137.3,136.8,132.2,129.8,129.3,128.6,127.4,126.2$, 125.7, 124.8, 124.1, 111.7, 111.4, 68.5, 68.0, 42.0, 41.7, 31.7, 31.3, 31.0, 29.5, 27.1, 26.1, 25.9, 22.8, 16.6, 14.2; FTIR $\left(\mathrm{cm}^{-1}\right)$ : $3020(\mathrm{w}$, aromatic and vinylic $\mathrm{C}-\mathrm{H}$ stretching), 2955, 2928, 2857 (w, aliphatic C-H stretching), 1687, 1602 ( $\mathrm{m}, \mathrm{C}=\mathrm{C}$ stretching), 1246 (s, C-O-C asymmetric stretching), 1016 ( $\mathrm{m}, \mathrm{C}-\mathrm{O}-\mathrm{C}$ symmetric stretching), 807 (s, aromatic $\mathrm{C}-\mathrm{H}$ out-of-plane bending), 967 ( $\mathrm{m}$, trans$\mathrm{HC}=\mathrm{CH}$ out-of-plane bending), $865(\mathrm{w}$, cis- $\mathrm{HC}=\mathrm{CH}$ outof-plane bending).

P3: $\mathrm{R}=$ dodecyl; aspect: brown paste; ${ }^{1} \mathrm{H} \quad \mathrm{NMR}$ (300 MHz, $\mathrm{CDCl}_{3}, \delta$ ): 7.80-6.40 (br m, aromatic and vinylic $\mathrm{H}), 4.10-3.80\left(\mathrm{~m}, \mathrm{OCH}_{2}\right), 2.21$ ( $\mathrm{s}, \mathrm{Ar}-\mathrm{CH}_{3}$ terminal group), 2.00-1.20 (br m, $-\left(\mathrm{CH}_{2}\right)_{10}-$ and $\left.\mathrm{C}\left(\mathrm{CH}_{3}\right)_{2}\right), 1.00-0.80(\mathrm{~m}$, $\left.\mathrm{CH}_{3}\right) ;{ }^{13} \mathrm{C}$ NMR $\left(75.5 \mathrm{MHz}, \mathrm{CDCl}_{3}, \delta\right): 155.0,154.7,142.9$, $142.6,137.3,137.2,132.3,132.2,129.6,129.3,128.9$, $128.7,128.6,127.6,126.8,126.4,126.2,125.7,124.8$, 124.2, 111.8, 111.4, 68.5, 68.0, 42.0, 41.7, 32.1, 31.3, 31.0, 29.8, 29.6, 29.5, 26.4, 26.2, 22.8, 16.7, 14.3; FTIR $\left(\mathrm{cm}^{-1}\right)$ : 3020 (w, aromatic and vinylic C-H stretching), 2922, 2852 (w, aliphatic C-H stretching), 1688, 1602 (m, C=C stretching), 1247 (s, C-O-C asymmetric stretching), 1026 (m, C-O-C symmetric stretching), 807 (s, aromatic $\mathrm{C}-\mathrm{H}$ out-of-plane bending), $967(\mathrm{~m}$, trans- $\mathrm{HC}=\mathrm{CH}$ out-of-plane bending), 865 ( $\mathrm{w}$, cis- $\mathrm{HC}=\mathrm{CH}$ out-of-plane bending).

P4: $\mathrm{R}$ = benzyl; aspect: pale yellow powder; ${ }^{1} \mathrm{H}$ NMR (300 MHz, $\mathrm{CDCl}_{3}, \delta$ ): 7.80-6.40 (br m, aromatic and vinylic $\mathrm{H}), 5.20-4.80\left(\mathrm{~m}, \mathrm{OCH}_{2}\right), 2.27$ ( $\mathrm{s}, \mathrm{Ar}-\mathrm{CH}_{3}$ terminal group), 1.85-1.40 (m, C $\left.\left(\mathrm{CH}_{3}\right)_{2}\right)$; ${ }^{13} \mathrm{C}$ NMR $\left(75.5 \mathrm{MHz} \mathrm{CDCl}_{3}, \delta\right)$ : $155.0,154.4,143.4,142.8,137.4,137.2,132.2,130.0$, $129.3,129.0,128.7,128.6,128.0,127.4,127.3,126.9$, 126.3, 124.9, 123.9, 112.4, 112.0, 110.8, 70.6, 70.4, 69.9, $42.1,42.9,41.8,31.3,31.0,16.8$; FTIR $\left(\mathrm{cm}^{-1}\right)$ : $3030(\mathrm{w}$, aromatic and vinylic C-H stretching), 2963, 2928, 2867 (w, aliphatic $\mathrm{C}-\mathrm{H}$ stretching), 1686, 1601 ( $\mathrm{m}, \mathrm{C}=\mathrm{C}$ stretching), 1244 (s, C-O-C asymmetric stretching), 1022 (m, C-O-C symmetric stretching), 808 (s, aromatic $\mathrm{C}-\mathrm{H}$ out-of-plane bending), 967 ( $\mathrm{m}$, trans $-\mathrm{HC}=\mathrm{CH}$ out-of-plane bending), 865 (w, cis-HC=CH out-of-plane bending). 


\subsection{Fabrication and characterization of diodes}

Single-layer devices were fabricated as sandwich structures between an aluminum ( $\mathrm{Al}$ ) cathode and an indium tin oxide (ITO) anode. The polymer solution $\left(10 \mathrm{mg} \mathrm{ml}^{-1}\right.$ in chloroform) was spin-cast (2000 rpm) onto ITO glass and the obtained film was then annealed at $40^{\circ} \mathrm{C}$ for $1 \mathrm{~h}$. The aluminum layer $(150 \mathrm{~nm})$ was deposited by thermal evaporation at $3.10^{-6}$ Torr. The current-voltage $(I-V)$ characteristics of the devices were recorded with a Keithley 236 source meter. Devices were fabricated and characterized in air at room temperature.

\section{Results and discussion}

\subsection{Synthesis and structural characterization}

The polymers P1-4 were synthesized from bisphenol A (BPA) according to a four-step synthetic route shown in Scheme 1. Four dichloromethyl aromatic derivatives (b1-4) were prepared by direct chloromethylation of the benzene ring of four different BPA ethers (a1-4), using the $\mathrm{HCl} /$ paraformaldehyde/acetic acid system and following a conventional procedure [25]. The corresponding di(triphenylphosphonium) salts (c1-4) were then obtained by acetone reflux of the dichloromethyl compounds in the presence of triphenylphosphine. The polymers were synthesized via the Wittig reaction by condensing the different di(triphenylphosphonium) salts with terephthaldicarboxaldehyde, according to a previously reported procedure using the $t$-BuOK/THF system [26]. Two heating procedures were tested: in Method A the polycondensation was carried out at room temperature for $24 \mathrm{~h}$, and in Method $\mathrm{B}$ the reaction was carried out initially at room temperature for $24 \mathrm{~h}$ and then the reaction mixture was heated to $60{ }^{\circ} \mathrm{C}$ at reflux for $4 \mathrm{~h}$. In both approaches the polymers were isolated and purified by precipitation in methanol. The polymer synthesis results are summarized in Table 1. An improvement in the reaction yield and the $D P_{\mathrm{n}}$ (number-average degree of polymerization) by increasing the aliphatic side-chain length was observed in both methods. Indeed, the incorporation of such alkoxy side-groups improves the solubility of the polymers in the reaction mixture, and the longer the aliphatic chains, the more significant their solubilizing effect. Comparison of the results of Methods A and B showed an improvement of the reaction yield and the polymer weight thanks to the sup- plementary heating step. For example, in the synthesis of P1, the yield increased from $60 \%$ to $76 \%$ and the polymer weight was doubled.

The obtained polymers were found to have good solubility in common organic solvents such as chloroform, dichloromethane, THF and toluene. The polymer structures were well established by ${ }^{1} \mathrm{H}$ NMR, ${ }^{13} \mathrm{C}$ NMR and FTIR spectroscopic analysis. The IR spectra showed the presence of both cis $\left(865 \mathrm{~cm}^{-1}\right)$ and trans $\left(967 \mathrm{~cm}^{-1}\right)$ vinylic absorptions [27]. In fact, according to their chemical structures, the yildes used in this work can be classified as semi-stabilized yildes, and their normal Wittig reaction with aldehydes is nonstereospecific and produces mixtures of cis and trans configurations nonstereospecifically [28]. The relative heights of the 865 and $967 \mathrm{~cm}^{-1}$ bands can be compared to give a quantitative analysis of the cis- and/ or trans-content of the sample. The cis-/trans-vinylene ratio of absorption coefficients of these two bands was estimated to be 0.46 by comparing the corresponding absorbencies in a similar model system, the cis,trans-1,4bis(4-ethoxystyryl)benzene (identified beforehand by ${ }^{1} \mathrm{H}$ NMR analysis). Thus, the cis-content of the polymer sample was calculated using the following equation: cis-content $(\%)=100 A_{\text {cis }} /\left(A_{\text {cis }}+0.57 A_{\text {trans }}\right)$, where $A_{\text {cis }}$ and $A_{\text {trans }}$ represent absorbencies of the 865 and $967 \mathrm{~cm}^{-1}$ bands, respectively. The obtained values were between $44 \%$ and $60 \%$ (Table 1).

The end-chain groups were identified using ${ }^{1} \mathrm{H}$ NMR analysis; the absence of aldehyde terminal groups was supported by the absence of the corresponding peak from the NMR spectra. On the other hand, the appearance of a weak signal at $2.10 \mathrm{ppm}$ in the ${ }^{1} \mathrm{H}$ NMR spectra and a peak at about $16.7 \mathrm{ppm}$ in the ${ }^{13} \mathrm{C}$ NMR spectra suggests toluene methyl terminal groups $\left(\mathrm{Ar}-\mathrm{CH}_{3}\right)$. This identification enabled us to determine the number-average molar weights of the polymers by comparing the integrations of the ${ }^{1} \mathrm{H}$ NMR signals corresponding to $\mathrm{Ar}-\mathrm{CH}_{3}$ groups and $\mathrm{OCH}_{2}$ units (Table 1).

\subsection{Optical properties}

The UV-vis absorption and photoluminescence spectra of the polymers P1-4 were recorded at room temperature, on dilute solutions in chloroform and on thin solid films. The absorption and fluorescence spectra of the polymer solutions were practically identical (Fig. 1), indicating no effect of the side-group structure on the conformation of the DSB moiety and, hence, on the effective conjugation

Table 1

Polymer synthesis results.

\begin{tabular}{|c|c|c|c|c|c|c|c|c|}
\hline & \multirow[t]{2}{*}{$\mathrm{R}$} & \multicolumn{3}{|c|}{ Method $A^{a}$} & \multicolumn{4}{|c|}{ Method B ${ }^{\mathrm{b}}$} \\
\hline & & Yield (\%) & $D P_{\mathrm{n}}{ }^{\mathrm{c}}$ & $M_{\mathrm{n}}\left(\mathrm{g} \mathrm{mol}^{-1}\right)$ & Yield (\%) & $D P_{\mathrm{n}}{ }^{\mathrm{c}}$ & $M_{\mathrm{n}}\left(\mathrm{g} \mathrm{mol}^{-1}\right)$ & Content of cis- $\mathrm{CH}=\mathrm{CH}(\%)$ \\
\hline P1 & Ethyl & 60 & 7 & 3180 & 76 & 14 & 6050 & 44 \\
\hline P2 & Hexyl & 67 & 14 & 7730 & 80 & 22 & 11,910 & 60 \\
\hline P3 & Dodecyl & 75 & 20 & 14,390 & 82 & 24 & 17,150 & 51 \\
\hline P4 & Benzyl & 62 & 11 & 6310 & 77 & 16 & 8980 & 45 \\
\hline
\end{tabular}

a Twenty-four hours at room temperature.

b Twenty-four hours at room temperature, then $4 \mathrm{~h}$ at reflux $\left(60^{\circ} \mathrm{C}\right)$.

c Determined by ${ }^{1} \mathrm{H}$ NMR analysis (by comparing the signal integration for toluene methyl terminal groups and $\mathrm{OCH}_{2}$ units). 


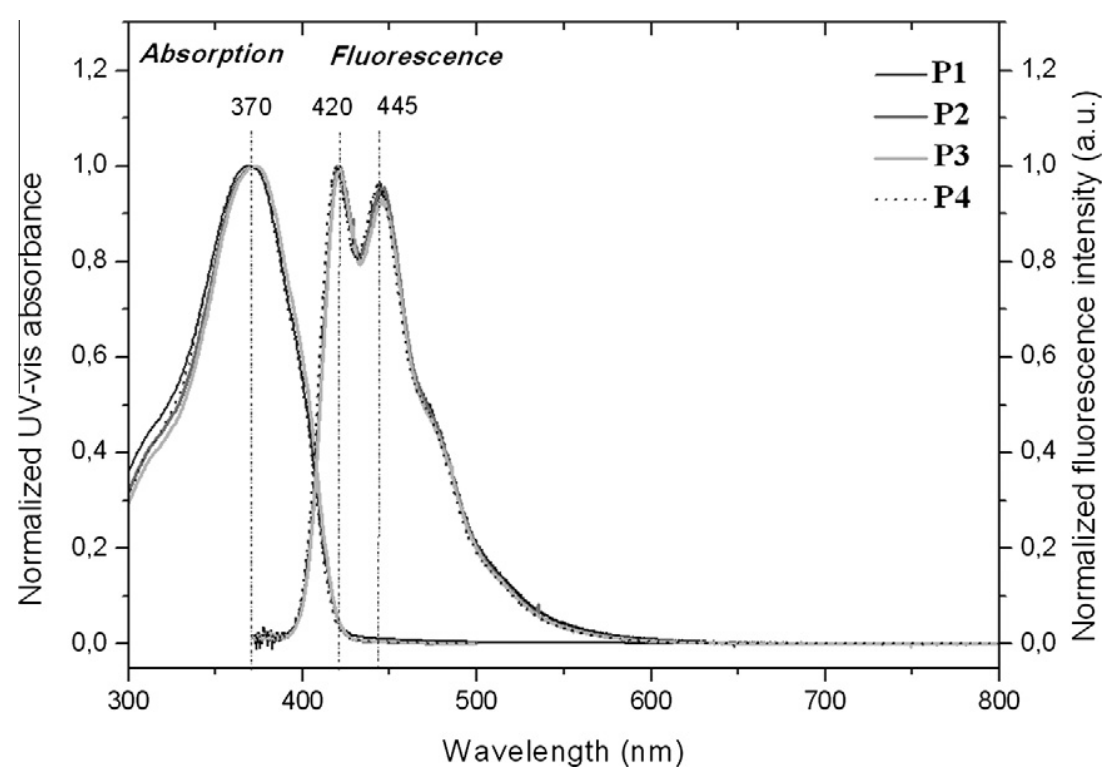

Fig. 1. UV-vis absorption and fluorescence spectra of polymers in chloroform $\left(5 \times 10^{-5}\right.$ and $2 \times 10^{-7} \mathrm{M}$ of DSB unit in absorption and in fluorescence, respectively).

length of the polymer in dilute solution. Such resemblance also indicates a negligible influence of the cis-/trans-vinylene ratio on the effective conjugation length. Indeed, the conjugated system (DSB) is relatively short and the range of the cis-content is sufficiently narrow (44-60\%) to considerably limit the effect of cis/trans ratio on the optical spectra of the polymers $[29,30]$. The absorption wavelength maxima are at about $370 \mathrm{~nm}$ and the absorption onsets at about $420 \mathrm{~nm}(2.95 \mathrm{eV})$. The absorption spectra are relatively broad and devoid of vibrational structure; this is a commonly observed feature in PPV-type chromophores, and is attributed to the wide range of vibrational and rotational levels available [31,32].

The polymer solutions exhibit a blue fluorescence and relatively narrow emission spectra with a FWHM (full width at half maximum) of about $60 \mathrm{~nm}$. Two well resolved emission bands at 420 and $445 \mathrm{~nm}$ and a shoulder around $478 \mathrm{~nm}$ were observed. The splitting of the emission spectra into two bands is in fact typical for PPV-type chromophores, which are characterized by a vibrational structure attributed to a coupling of the phenylene ring stretching modes with the $\pi-\pi$ electronic transitions [33]. The fluorescence quantum efficiencies of the polymers were determined in dilute chloroform by a relative method using quinine sulfate as standard [34]. The values obtained were $44 \%, 47 \%, 28 \%$ and $58 \%$ for P1, P2, P3 and P4, respectively.

In the solid film state, the polymer absorption profiles are qualitatively similar (Fig. 2). Nevertheless, a difference in the long-wavelength tails was observed and a significantly weaker absorbance was found for $\mathbf{P} \mathbf{3}$ in this region. In fact, $\pi-\pi$ interaction between parallel conjugated segments can generally take place in semi-conducting polymer films, forming aggregates which absorb at high wavelengths and red-shift the absorption onset compared to the corresponding polymer solution. Thus, the UV-vis spectra obtained confirm the existence of such an interaction in the films of P1, P2 and P4, since higher absorption onsets were found compared to the solution ones $\left(\lambda_{\text {onset }}\right.$, Table 2). On the other hand, in the case of P3, the film absorption onset is almost equal to that in solution, indicating an effective suppression of the $\pi-\pi$ interaction by the incorporation of bulky dodecyl side-chains on the distyrylbenzene moiety. The optical band gaps estimated from the absorption onset of the polymers films are 2.80 , 2.87, 2.94 and $2.90 \mathrm{eV}$ for P1, P2, P3 and P4, respectively.

The fluorescence spectra of the polymers films present significant differences in the profile and mainly in the spectrum width (Fig. 2). The emission spectra of P2, P3 and $\mathbf{P 4}$ lie in the blue region and consist of two maxima towards 425 and $450 \mathrm{~nm}$ and a shoulder at about $483 \mathrm{~nm}$. The P1 spectrum shows the same two maxima (at 428 and $453 \mathrm{~nm}$ ), but the shoulder is transformed into a discrete band and a new shoulder appears at higher wavelengths; a broad spectrum was obtained $(161 \mathrm{~nm})$ and a green emission found for $\mathbf{P 1}$ film. The spectrum width decreases then upon going to the hexylated polymer P2 $(88 \mathrm{~nm})$, and even more upon going to the benzylated polymer $\mathbf{P 4}(84 \mathrm{~nm})$. The narrowest spectrum is obtained for polymer P3, incorporating the dodecyloxy chains $(72 \mathrm{~nm})$. There is, therefore, a clear correlation between the length of the aliphatic side-chain and the FWHM value and the spectrum width decreases with increase in the chain length. We also noticed a reduction in the relative intensity of the shoulder towards $483 \mathrm{~nm}$ in favor of that of the first band $(425 \mathrm{~nm})$ in the same order, i.e. P1, P2, P3. Such behavior is mainly related to the interaction of the distyrylbenzene units in the condensed phase. Depending on the distance, chromophore interactions give rise to new red-shifted electronic states. In the absorption spectrum, they are visible as featureless absorption tails. Furthermore they reabsorb the emission of isolated 


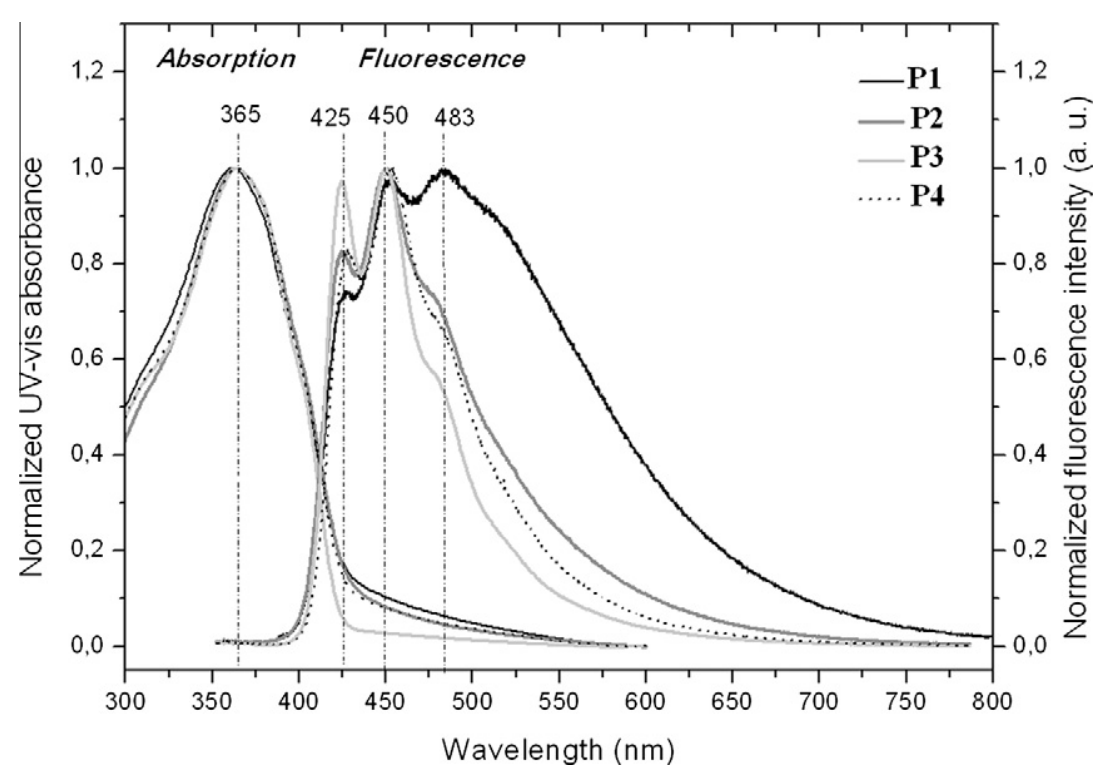

Fig. 2. UV-vis absorption and fluorescence spectra of polymers in thin solid film (40-70 nm).

Table 2

Optical data for polymers in thin solid films.

\begin{tabular}{lllllll}
\hline & \multicolumn{3}{l}{ Absorption } & & Fluorescence \\
\cline { 2 - 3 } \cline { 6 - 7 } & $\begin{array}{l}\lambda_{\max } \\
(\mathrm{nm})\end{array}$ & $\begin{array}{l}\varepsilon_{\max } \\
\left(10^{4} \mathrm{~cm}^{-1}\right)\end{array}$ & $\begin{array}{l}\lambda_{\text {onset }} \\
(\mathrm{nm})\end{array}$ & $\begin{array}{l}\lambda_{\max } \\
(\mathrm{nm})\end{array}$ & $\begin{array}{l}\mathrm{FWHM}^{\mathrm{b}} \\
(\mathrm{nm})\end{array}$ \\
\hline P1 & 364 & 7.78 & 443 & & $428 ; 453 ; 484 ; 510^{\mathrm{a}}$ & 161 \\
P2 & 364 & 9.79 & 433 & & $425 ; 450 ; 478^{\mathrm{a}}$ & 88 \\
P3 & 364 & 5.98 & 422 & & $424 ; 450 ; 478^{\mathrm{a}}$ & 72 \\
P4 & 362 & 5.91 & 428 & & $428 ; 453 ; 478^{\mathrm{a}}$ & 84 \\
\hline
\end{tabular}

a Shoulder.

b Full width half maximum of the spectrum.

chromophores, decreasing their $0-0$ emission peak. The emission band of the isolated chromophores and the absorption band of aggregated chromophores correspond, so a significant energy transfer occurs and brings about the broad low-energy emission bands. To conclude, the more bulky the side-group, the more hindered the $\pi-\pi$ interactions and, therefore, the narrower the emission spectrum. According to this reasoning and by comparing the emission spectra profiles (especially the FWHM values), we can also conclude that the benzyl group presents an effect comparable to that exhibited by the hexyl chain on the $\pi-\pi$ interactions in polymer solid films.

\subsection{HOMO and LUMO energy levels}

Cyclic voltammetry (CV) was employed to estimate the HOMO (Highest Occupied Molecular Orbital) and LUMO (Lowest Unoccupied Molecular Orbital) energy levels of the polymers. These molecular parameters are generally used as synonyms of the top of the valence band or ionization potential, and of the bottom of the conduction band or electron affinity, respectively. In fact, knowledge of these energy levels is of crucial importance to determine energy barriers and select cathode and anode materials for PLEDs [35]. These parameters are also of essential importance in optimizing the efficiency of charge-carrier photogeneration in polymeric solar cells, and charge injection in polymeric thin-film transistors [36]. CV analysis is reliable, as the electrochemical processes are similar to those involved in charge injection and transport processes in such electronic devices [37].

The polymers films were drop-coated onto ITO glass substrates and scanned both at positive and negative potentials in a $0.1 \mathrm{M}(\mathrm{n}-\mathrm{Bu})_{4} \mathrm{NClO}_{4} /$ acetonitrile solution at a scan rate of $50 \mathrm{mV} \mathrm{s}^{-1}$. According to an empirical method, and by assuming that the energy level of the ferrocene/ferrocenium is $4.8 \mathrm{eV}$ below the vacuum level, the HOMO energy level, LUMO energy level and the electrochemical gap $\left(E_{\text {g-el }}\right)$ can be calculated as follows [38]:

$E_{\text {Hомо }}(\mathrm{IP}$, ionization potential $)=-\left(V_{\text {onset-ox }}-V_{\mathrm{FOC}}+4.8\right) \mathrm{eV}$ $E_{\text {LUMo }}(\mathrm{EA}$, electron affinity $)=-\left(V_{\text {onset-red }}-V_{\text {FoC }}+4.8\right) \mathrm{eV}$ $E_{\text {g-el }}=\left(E_{\text {LUMO }}-E_{\text {Hомо }}\right) \mathrm{eV}$

where, $V_{\mathrm{FOC}}$ is the ferrocene half-wave potential $(0.55 \mathrm{~V})$, $V_{\text {onset-ox }}$ is the polymer oxidation onset and $V_{\text {onset-red }}$ is the polymer reduction onset, all measured versus SCE. The estimated $E_{\text {номо, }} E_{\mathrm{LUMO}}$ and $E_{\text {g-el }}$ values are summarized in Table 3. These values, characteristic of the electronic conjugation in oligo-PPV moieties [39], could be affected mainly by torsions in the conjugated system, and to a much lesser extent by the polymer environment. Indeed for the four compounds they are similar enough to ascertain the rigidity of the conjugated segment. As observed for the optical gaps, the narrowest electrochemical gap is obtained in the case of P1, and the broadest for P3; P2 and P4 present comparable gaps. However, a slight discrepancy is observed between the band gaps obtained by the optical method $(2.80-2.95 \mathrm{eV})$ and by electrochemical analysis 
Table 3

Electrochemical data for polymers P1-4

\begin{tabular}{llllll}
\hline & $\begin{array}{l}V_{\text {onset-ox }} \\
(\mathrm{V})\end{array}$ & $\begin{array}{l}V_{\text {onset-red }} \\
(\mathrm{V})\end{array}$ & $\begin{array}{l}E_{\text {HOMO }} \\
(\mathrm{eV})\end{array}$ & $\begin{array}{l}E_{\mathrm{LUMO}} \\
(\mathrm{eV})\end{array}$ & $\begin{array}{l}E_{\text {g-el }} \\
(\mathrm{eV})\end{array}$ \\
\hline P1 & 1.06 & -1.93 & -5.31 & -2.32 & 2.99 \\
P2 & 1.11 & -1.96 & -5.36 & -2.29 & 3.07 \\
P3 & 1.17 & -1.98 & -5.42 & -2.27 & 3.15 \\
P4 & 1.09 & -1.97 & -5.34 & -2.28 & 3.06 \\
\hline
\end{tabular}

(3.00-3.15 eV). Such a difference has been previously reported in conjugated polymers and was attributed to the interface barrier between the polymer film and the electrode surface [40]. Nevertheless, it must be pointed out that the gap values obtained by electrochemistry should not be compared directly with the optical gaps but rather with the electrical ones and furthermore the measured electrochemical gap values are affected by solvation, so they are underestimated relative to the electrical gap. On the other hand, optical gaps characterize the excited states and their values are also smaller than the electrical ones, by the exciton binding energies [41]. The similarity of the values found experimentally for electrochemical and optical gaps shows that solvation and exciton state binding energies must be of the same magnitude, probably around $1 \mathrm{eV}$ [42], which is a reasonable value, in view the size of the conjugated electronic system. The electrical gap then would be about $4 \mathrm{eV}$.

\subsection{Electrical characterization}

Single-layer devices with the [ITO/polymer/Al] configuration were fabricated to investigate the current-voltage $(I-V)$ characteristics of the polymers P1-4. As shown in Fig. 3, the $I-V$ curves indicate different behaviors with relatively low turn-on voltages of 2.6, 2.7, 4.9 and $3.8 \mathrm{~V}$ for P1, P2, P3 and P4, respectively. From the sizes of the pendant group, it can be inferred that interchain electron transfer is the most favorable in the ethylated polymer P1, and the least in polymer $\mathbf{P 3}$ incorporating the dodecyl side-chains. Indeed, preliminary analysis indicates than the $I-V$ characteristic for P1 corresponds effectively to a diode behavior, dominated by injection from electrodes. For P2, P3 and $\mathbf{P 4}$, the shapes correspond to a current limitation by space charges, due to the lower carrier mobility. The discrepancy in the turn-on voltage may be due the difference in the $\pi-\pi$ interaction between the conjugated segments in the solid state. Such interaction tends, in fact, to promote good carrier transport through the polymer film. However, more detailed studies are underway to understand the disparity in the turn-on voltage, which may be due also to the morphological features of the films.
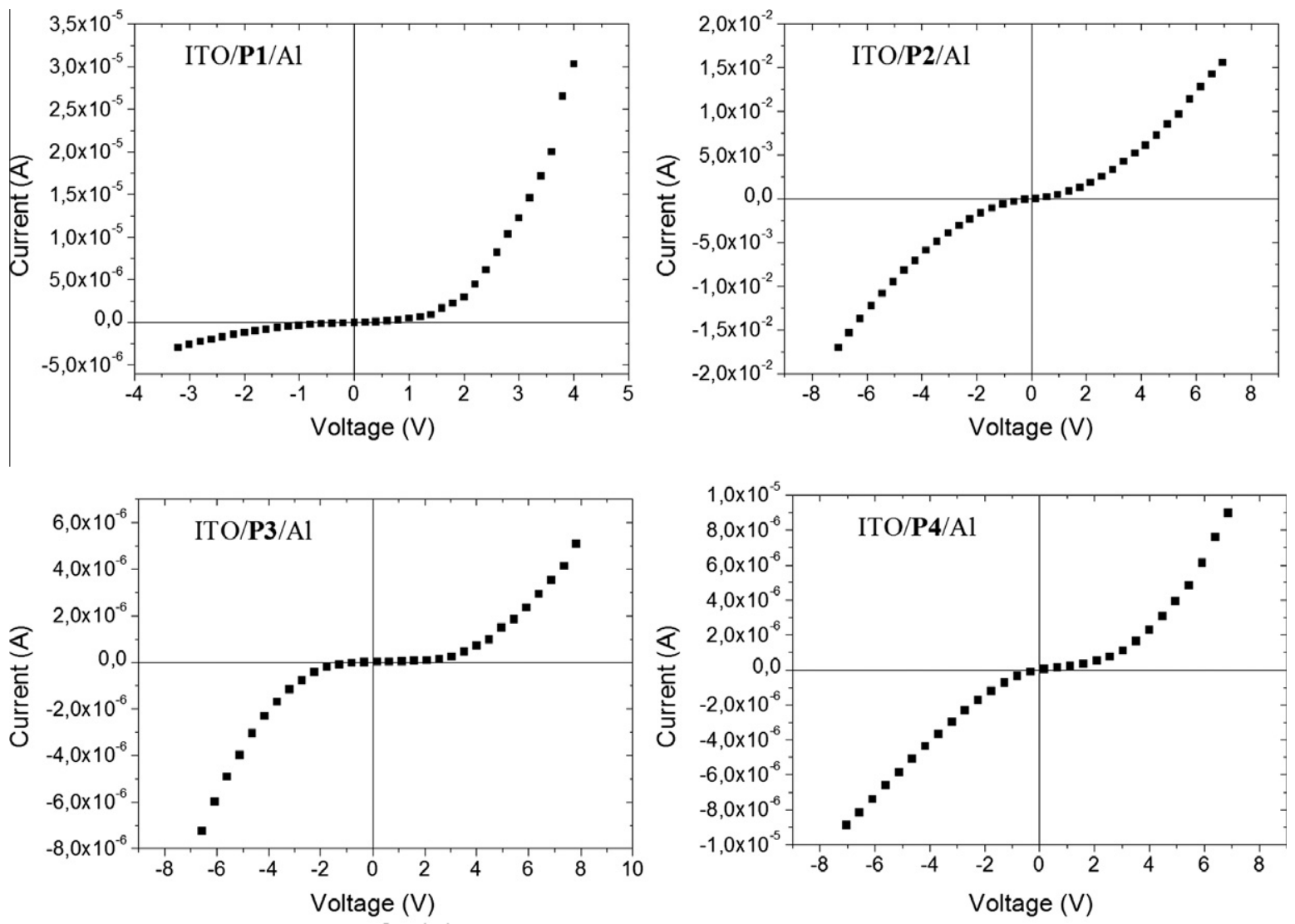

Fig. 3. Current-voltage curves of [ITO/polymer/Al] devices. 


\section{Conclusion}

We have synthesized and characterized a series of semiconducting polymers derived from bisphenol A and containing separated substituted distyrylbenzenes (DSB) as uniform conjugated moieties. Various side-groups were attached to the DSB segments: linear alkyloxy chains of different lengths (ethyloxy: P1, hexyloxy: P2, dodecyloxy: P3) and the benzyloxy group (P4). Study of the optical properties of P1-4 in dilute solution shows a blue emission and practically identical emission spectra characteristic of isolated DSB for all polymers. In contrast, in the solid state, a $\pi-\pi$ interaction between the DSB segments can occur and control the photophysical behavior of the polymer, depending on the side-group structure. Indeed, there is a clear correlation between the aliphatic side-chain length and the optical properties of polymer thin films: the longer the chain, the larger the gap and the narrower the emission spectrum. In spite of the conjugation confinement in a short PPV segment (DSB), the results show that is impossible to obtain blue fluorescence in the ethylated polymer (P1) film. The introduction of dodecyl (P3) or even hexyl (P2) chains is enough to prevent inter-DSB $\pi-\pi$ stacking and to acquire the blue emission. The effect of the benzyl group on the solid-state $\pi-\pi$ interaction is comparable to that of the hexyl group, but the benzylated polymer $\mathbf{P 4}$ has a higher fluorescence quantum efficiency. Concerning the electrical behavior, low turn-on voltages between 2.6 and $4.9 \mathrm{~V}$ were obtained for single-layer devices of the [ITO/polymer/Al] configuration.

\section{Acknowledgements}

This work was financially supported by the Ministry of Higher Education and Scientific Research of Tunisia and the contract CMCU No. 05S1304. The authors thank Dr. Rafik Ben Chaâbane and Mrs. Maha Benzarti-Ghédira (LPCI, Faculty of Sciences, Monastir) for the electrical measurements and Dr. Carlos Barthou (INSP, Université Paris 6) for the photoluminescence analysis. Special thanks go to Dr. John Lomas (ITODYS, Université Paris 7) for his help to improve the English quality of the manuscript and for his scientific remarks.

\section{References}

[1] Burroughes JH, Bradley DDC, Brown AR, Marks RN, Mackay K, Friend $\mathrm{RH}$, et al. Light-emitting diodes based on conjugated polymers. Nature 1990;347:539-41.

[2] Kraft A, Grimsdale AC, Holmes AB. Electroluminescent conjugated polymers - seeing polymers in a new light. Angew Chem Int Ed Engl 1998;37:402-28.

[3] Akcelrud L. Electroluminescent polymers. Prog Polym Sci 2003;28: 875-962.

[4] Segura JL. The chemistry of electroluminescent organic materials. Acta Polym 1998;49:319-44.

[5] Xiang D, Shen Q, Zhang S, Jiang X. Synthesis and characterization of novel soluble alternating copoly(phenylene vinylene) derivative for light-emitting electrochemical cell. J Appl Polym Sci 2003;88: 1350-6.

[6] Sun Q, Yang C, Wang H, He G, Li Y. Polymer light-emitting electrochemical cell based on a block copolymer containing tri(ethyleneoxide) spacers. Polym Adv Technol 2002;13:663-9.

[7] Murphy AR, Fréchet JMJ. Organic semiconducting oligomers for use in thin film transistors. Chem Rev 2007;107:1066-96.
[8] Gunes S, Neugebauer H, Sariciftci NS. Conjugated polymer-based organic solar cells. Chem Rev 2007;107:1324-38.

[9] Segura JL, Martin N, Guldi DM. Materials for organic solar cells: the C60/ $\pi$-conjugated oligomer approach. Chem Soc Rev 2005;34: $31-47$.

[10] Thomas III SW, Joly GD, Swager TM. Chemical sensors based on amplifying fluorescent conjugated polymers. Chem Rev 2007;107: 1339-86.

[11] Lange U, Roznyatovskaya NV, Mirsky VM. Conducting polymers in chemical sensors and arrays. Anal Chim Acta 2008;614:1-26.

[12] Kim DY, Cho HN, Kim CY. Blue light emitting polymers. Prog Polym Sci 2000;25:1089-139.

[13] Ohmori Y, Uchida K, Muro K, Yoshino K. Blue electroluminescent diodes utilizing poly(alkylfluorene). Jpn J Appl Phys 1991;30: 1941-3.

[14] Lee JH, Hwang DH. Alkoxyphenyl-substituted polyfluorene: a stable blue-light-emitting polymer with good solution processability. Chem Commun 2003;22:2836-7.

[15] Edwards A, Blumstengel S, Sokolik I, Dorsinville R, Yun H, Kwei TK, et al. Blue photo- and electroluminescence from poly(benzoyl-1,4phenylene). Appl Phys Lett 1997;70:298-300.

[16] Andersson MR, Berggren M, Gustafsson G, Hjertberg T, Inganäs $O$, Wennerström O. Synthesis of poly(alkylthiophenes) for lightemitting diodes. Synth Met 1995;71:2183-4.

[17] Liao L, Pang Y, Ding L, Karasz FE. Blue-emitting soluble poly $(m-$ phenylenevinylene) derivatives. Macromolecules 2001;34:7300-5.

[18] Brown AR, Burn PL, Bradley DDC, Friend RH, Kraft A, Holmes AB. Blue-shifted electroluminescence from a stable precursor to $\operatorname{poly}(p-$ phenylene vinylene). Mol Cryst Liq Cryst 1992;216:111-6.

[19] Gowri R, Mandal D, Shivkumar B, Ramakrishnan S. Synthesis of novel poly[(2,5-dimethoxy-p-phenylene)vinylene] precursors having two eliminatable groups: an approach for the control of conjugation length. Macromolecules 1998;31:1819-26.

[20] Hay M, Klavetter FL. Aliphatic phenylene vinylene copolymers: tuning the color of luminescence through co-monomer feed ratios. J Am Chem Soc 1995;117:7112-8.

[21] Yang Z, Sokolik I, Karasz FE. A soluble blue-light-emitting polymer. Macromolecules 1993;26:1188-90.

[22] Liau CY, Gan YY, Zhou Y, Lam YL, Gan LH. Studies of an intrinsically soluble copolymer with well-defined alternating substituted $p$ phenylenevinylene and hexanedioxy blocks. Polymer 2000;41: 7339-46.

[23] Rothberg LJ, Yan M, Papadimitrakopoulos F, Galvin ME, Kwock EW, Miller TM. Photophysics of phenylenevinylene polymers. Synth Met 1996;80:41-58.

[24] Jaballah N, Trad H, Majdoub M, Jouini M, Roussel J, Fave JL. Synthesis and characterization of new blue-photoluminescent copolymer derived from bisphenol A. J Appl Polym Sci 2006;99:2997-3004.

[25] Trad H, Majdoub M, Davenas J. Soluble PPVs with few structural defects: synthesis and characterization. Mater Sci Eng C 2006;26: 334-9.

[26] Jaballah N, Majdoub M, Fave JL, Barthou C, Jouini M, Tanguy J. New confined $p$-phenylenevinylene (PPV)-type polymer analogue of poly(phenylene sulfide). Eur Polym J 2008;44:2886-92.

[27] Wang F, He F, Xie Z, Li M, Hanif M, Gu X, et al. A solution-processible poly( $p$-phenylene vinylene) without alkyl substitution: introducing the cis-vinylene segments in polymer chain for improved solubility, blue emission, and high efficiency. J Polym Sci, Part A: Polym Chem 2008;46:5242-50.

[28] Ndayikengurukiye H, Jacobs S, Tachelet W, Van Der Looy J, Pollaris A, Geise HJ, et al. Alkoxylated p-phenylenevinylene oligomers: synthesis and spectroscopic and electrochemical properties. Tetrahedron 1997;53:13811-28.

[29] Wang F, He F, Xie ZQ, Li YP, Hanif M, Li M, et al. Poly(p-phenylene vinylene) derivatives with different contents of cis-olefins and their effect on the optical properties. Macromol Chem Phys 2008;209: 1381-8.

[30] Liao L, Ding L, Karasz FE, Pang Y. Poly[(2-alkoxy-5-methyl-1,3phenylene vinylene)-alt-(phenylene vinylene)] derivatives with different contents of cis- and trans-olefins: the effect of the olefin bond geometry and conjugation length on luminescence. J Polym Sci, Part A: Polym Chem 2004;42:303-16.

[31] Ichino Y, Ni JP, Ueda Y, Wang DK. Vibronic structures in the optical spectra of distyrylbenzene derivatives. Synth Met 2001;116:223-7.

[32] Henari FZ, Manaa H, Kretsch KP, Blau WJ, Rost H, Pfeiffer S, et al. Effective stimulated emission and excited state absorption measurements in the phenylene-vinylene oligomer (1,4-bis-(alphacyanostyryl)-2,5-dimethoxybenzene). Chem Phys Lett 1999;307: 163-6. 
[33] Ton-That C, Stockton G, Phillips MR, Nguyen TP, Huang CH, Cojocaru A. Luminescence properties of poly (phenylene vinylene) derivatives. Polym Int 2008;57:496-501.

[34] Feng L, Chen Z. Synthesis and photoluminescent properties of polymer containing perylene and fluorene units. Polymer 2005;46: 3952-6.

[35] Fan B, Sun Q Song N, Wang H, Fan H, Li Y. Electroluminescent properties of a partially-conjugated hyperbranched $\operatorname{poly}(p-$ phenylene vinylene). Polym Adv Technol 2006;17:145-9.

[36] Shirota Y, Kageyama H. Charge carrier transporting molecular materials and their applications in devices. Chem Rev 2007:107: 953-1010.

[37] Cheng M, Xiao Y, Yu WL, Chen ZK, Lai YH, Huang W. Synthesis and characterization of a cyano-substituted electroluminescent polymer with well-defined conjugation length. Thin Solid Films 2000;363: 110-3.

[38] Bredas JL, Silbey R, Bordeaux DS, Chance RR. Chain-length dependence of electronic and electrochemical properties of conjugated systems: polyacetylene, polyphenylene, polythiophene, and polypyrrole. J Am Chem Soc 1983;105:6555-9.

[39] Wu F, Tian W, Sun J, Shen J, Pan X, Su Z. Study on the electronic structure of phenylene vinylene dimers with different substituents. Mater Sci Eng B 2001;85:165-8.

[40] Egbe DAM, Carbonnier B, Paul EL, Muhlbacher D, Kietzke T, Birckner $\mathrm{E}$, et al. Diyne-containing PPVs: solid-state properties and comparison of their photophysical and electrochemical properties with those of their yne-containing counterparts. Macromolecules 2005;38:6269-75.

[41] Zahn DRT, Gavrila GN, Salvan G. Electronic and vibrational spectroscopies applied to organic/inorganic interfaces. Chem Rev 2007;107:1161-232.

[42] Djurovich PI, Mayo EI, Forrest SR, Thompson ME. Measurement of the lowest unoccupied molecular orbital energies of molecular organic semiconductors. Org Electron 2009;10:515-20. 